\title{
LES GRANDS NEVROSES DE L'INTELLIGENCE
}

\author{
Jorge Ramos \\ Do Instituto de Cultura Americana - Lisboa
}

Si la personnalité et les caractères forment le style et les idées, c'est-à-dire le procédé artistique et la vision des choses, on est bien obligé d'admettre aussi que les grands troubles nerveux, les déséquilibres psychiques et parfois les stigmates héréditaires ont une influence sur les oeuvres d'imagination.

Les biographies complètes et documentées des grands écrivains composent une impressionnante collection de maladies nerveuses. L'intelligence créatrice, déjà fait anormal en soi-même, révèle, lorsqu'elle atteint le summum du génie, un état particulier qui intéresse non seulement la psychologie mais aussi la curiosité scientifique. La médecine possède dans la littérature un énorme laboratoire de recherches. Le cas exceptionnel du génie porte avec lui l'empreinte d'un complexe psychique auquel ne peuvent être étrangers certains écarts de l'équilibre naturel de l'homme. L'influence pathologique est manifeste dans l'oeuvre d'art.

Proust, auteur d'une oeuvre immense au sentiment obscur se complaisant dans l'analyse des choses, dans l'association des sensations et des pensées (cf. la composition touffue de ses livres comme par exemple Sodome et Gomorrhe où une seule phrase occupe trois pages), était d'une sensibilité anormale, peut-être du même type névrosé que Rousseau, un asthmatique depuis son adolescence, obsédé par l'idée de mourir subitement, un hyperémotif concentré sur lui-même, un auto-analyste vivant son propre drame dans la littérature. Tout au long de l'échelle psycho-esthétique de son oeuvre apparait le schizoïde et selon Delacroix: "on peut faire d'une pointe de schizoïdie un instrument de dé- 
couverte psychologique". Homme du monde extérieur et des sentiments, il vécut toujours sous l'oppression de son infirmité. "Je ne sais rien du monde et c'est en restant immobile comme un escabeau que je vois seulement un peu dans les ténèbres". Chez lui, le don d'observation ou mieux, de discerner les détails ne pouvait être plus extraordinaire. Dans les pages touffues et les longues périodes de Du côté de chez Swann, on devine un esprit tourmenté qui se réfugie dans l'un des plus grands isolements - comme s'il n'avait pas été de ce monde, un homme qui se promenait en dandy dans les salons élégants de Paris, où, admirable collectionneur d'insectes d'ailleurs, il cueillait dans chaque geste, dans chaque phrase, dans chaque attitude, dans les moindres détails, les éléments qui forment la structure d'une gigantesque oeuvre d'analyse de la société de son temps: frivole, oisive et crevant d'ennui.

C'est un psycho-timide qui promène son désoeuvrement dans les salons aristocratiques de Paris, méritant cette définition de Barrès: "un prince persan égaré dans une loge de concierge", et qu'Anatole France, sans ambages, confessa n'avoir jamais compris comme écrivain. La littérature fut le palliatif antispasmodique qui éloigna ou atténua ses crises.

Un autre cas, celui d'Edgard Poe - cas morbide, s'il en est, d'une imagination hallucinée. Poe fut un génie. Et comme tous les génies, il fut d'une étonnante clairvoyance. Au travers d'une sensibilité aiguisée par la névrose, il s'est aperçu que ce n'est pas avec le regard banal des autres qu'on pouvait voir.

Comme Proust, Edgard Poe a vécu dans l'enfer de sa pensée. Il dirigea simplement son imagination de l'autre côté sombre de la vie. Le tempérament morbide, maladif de Proust, le poussa à accepter la vie comme une humiliation qu'il est nécessaire de vaincre. Il la hait mais l'oeuvre qu'il nous a laissée a une tragique saveur d'odyssée, un sentiment presque viril, héroïque, dirai-je. Ce que la vie a d'amer et d'angoissant apparaît en demi-teintes; voilé par un inconformisme mais se bornant à une sorte de résigna. tion douloureuse. Chez Poe, l'homme et le monde sont des visions qu'on dirait arrachées à l'enfer . . . II n'y a pas d'aquarelles subtiles, mais de grands cadres. Poe est le prosateur de l'épou- 
vante de la peur, et son art renferme la poésie du tragique dans sa plus haute expression atteignant les cimes culminantes de l'impressionnant. C'est avec des frissons de terreur, avec des notes absurdes arrachées au clavier de l'extra-humain, c'est avec des images caverneuses de Délire, avec d'étranges visions qui ont quelque chose de sépulchral pour la vie même, qu'il a édifié le monument de son oeuvre immortelle.

Le fantastique de Poe est plus terrible que celui d'Hoffmann et l'a rendu célèbre avec ces chefs d'oeuvres que sont Le Corbeau, Le Silence, Le Scarabée d'Or, Le Chat Noir. Maupassant dans Le Horla fait figure de disciple. Baudelaire semble être conduit par la main spectrale de l'écrivain qui a écrit La Chute de la Maison Usher. Les plus invraisemblables créations de Wells, les névroses de Rollinat, les cauchiemars de Kipling se ressentent de l'influence de l'auteur du Puits et du Pendule, où le mystérieux et le terrifiant atteignent leur point culminant. Le monde tel qu'il l'imagina, est un monde où les morts vivent encore sous terre, où les agonies sont lentes comme dans les hôpitaux, où la Mort n'est qu'une lente décomposition ou une énigme de la Fatalité.

II y a aujourd'hui 105 ans que les cruelles insomnies de Poe ont trouvé le repos de la nuit éternelle - la Mort qui rugissait vengeresse et sinistre dans Hop-Frog, qui semblait visqueuse et lente dans Le Masque de la Mort Rouge, qui se cachait dans les orbites vides du Roi Peste et dans le manteau diaphane de Bérénice, ou que nous devinons, comme une fantasmagorie de minuit. sur les pas silencieux ou dans le mutisme glacé de Ligêia.

L'hypersensibilité de Stendhal commande toute son oeuvre qui nous surprend par sa passion à éplucher le subconscient comme si cela résultait du désir de fixer le choc des impulsions et des sentiments.

Tolstoï, "sensibilité impulsive sous les dehors d'un théoricien inflexible", comme le définit Halperine Taminsky dans la préface des Lettres Inédites de Tolstoï, dans son esquisse d'un cadre nouveau de la personnalité humaine, dans la description des types des visages et des évènements comme historien de coutu- 
mes et créateur de symboles, nous montre sans peine les syndromes tempéramentaux de sa morbidité, cultivée dans l'excitation de son monde affectif - où il y a une force passionnelle de pénétration semblable à celle des microscopiques molécules de radium dans le champ de la physique... L'individualisme de Tolstoï (visible par les allégories frappantes qui reflètent un pouvoir non commun pour exprimer les idées. II fut un nerveux, voyant dans l'activité et la lutte l'origine de tout le mal, ex. La Guerre et la Paix) atteint largement l'esprit universel.

Le processus de composition littéraire d'André Gide ou de Rémy de Gourmont, riche en subtilités d'analyses psychologiques, correspond à une espèce de renoncement et d'apathie constatée fréquemment chez les hommes de génie. Et pour être le génie porteur de névroses, ces états morbides ont pour ainsi dire le pouvoir de révéler les plus surprenantes perspicacités de l'esprit... 